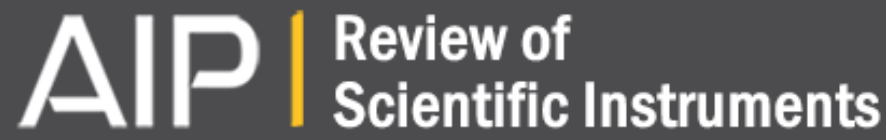

\section{Magnetic tweezers with high permeability electromagnets for fast actuation of magnetic beads}

La Chen, Andreas Offenhäusser, and Hans-Joachim Krause

Citation: Review of Scientific Instruments 86, 044701 (2015); doi: 10.1063/1.4916255

View online: http://dx.doi.org/10.1063/1.4916255

View Table of Contents: http://scitation.aip.org/content/aip/journal/rsi/86/4?ver=pdfcov

Published by the AIP Publishing

\section{Articles you may be interested in}

Fine-tuning of magnetic and microfluidic viscous forces for specific magnetic bead-based immunocomplex formation

J. Appl. Phys. 114, 064903 (2013); 10.1063/1.4817663

Magnet polepiece design for uniform magnetic force on superparamagnetic beads

Rev. Sci. Instrum. 81, 074303 (2010); 10.1063/1.3469792

Enhancement of radiated noise suppression on flexible magnetic thick film electromagnetic interference filters with high-frequency permeability on data signal cable

J. Appl. Phys. 107, 09A509 (2010); 10.1063/1.3356234

Quadrupolar magnetic actuation of superparamagnetic particles for enhanced microfluidic perfusion Appl. Phys. Lett. 94, 022505 (2009); 10.1063/1.3072592

A fast algorithm for nonlinear finite element analysis using equivalent magnetization current J. Appl. Phys. 91, 8718 (2002); 10.1063/1.1456394

Frustrated by

old technology?

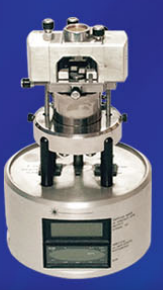

Is your AFM dead and can't be repaired?

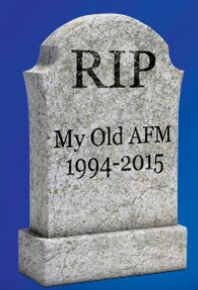

Sick of bad customer support?

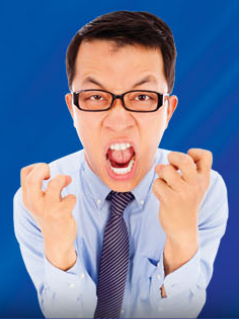

It is time to upgrade your AFM

Minimum $\$ 20,000$ trade-in discount

for purchases before August 31st

Asylum Research is today's technology leader in AFM 


\title{
Magnetic tweezers with high permeability electromagnets for fast actuation of magnetic beads
}

\author{
La Chen, Andreas Offenhäusser, and Hans-Joachim Krause ${ }^{a)}$ \\ Institute of Bioelectronics (ICS-8/PGI-8), Forschungszentrum Jülich GmbH, 52425 Jülich, Germany
}

(Received 19 November 2014; accepted 13 March 2015; published online 1 April 2015)

\begin{abstract}
As a powerful and versatile scientific instrument, magnetic tweezers have been widely used in biophysical research areas, such as mechanical cell properties and single molecule manipulation. If one wants to steer bead position, the nonlinearity of magnetic properties and the strong position dependence of the magnetic field in most magnetic tweezers lead to quite a challenge in their control. In this article, we report multi-pole electromagnetic tweezers with high permeability cores yielding high force output, good maneuverability, and flexible design. For modeling, we adopted a piece-wise linear dependence of magnetization on field to characterize the magnetic beads. We implemented a bi-linear interpolation of magnetic field in the work space, based on a lookup table obtained from finite element simulation. The electronics and software were custom-made to achieve high performance. In addition, the effects of dimension and defect on structure of magnetic tips also were inspected. In a workspace with size of $0.1 \times 0.1 \mathrm{~mm}^{2}$, a force of up to $400 \mathrm{pN}$ can be applied on a $2.8 \mu \mathrm{m}$ superparamagnetic bead in any direction within the plane. Because the magnetic particle is always pulled towards a tip, the pulling forces from the pole tips have to be well balanced in order to achieve control of the particle's position. Active video tracking based feedback control is implemented, which is able to work at a speed of up to $1 \mathrm{kHz}$, yielding good maneuverability of the magnetic beads. (C) 2015 AIP Publishing LLC. [http://dx.doi.org/10.1063/1.4916255]
\end{abstract}

\section{INTRODUCTION}

There are numerous techniques for characterizing force properties in biology. Optical tweezers, atomic force microscopy (AFM), and magnetic tweezers are the most commonly used tools. Magnetic tweezers exhibit a number of advantages, for example, relatively high force (compared to optical tweezers), multi-dimensional actuation, and high spatial and temporal resolution. ${ }^{1}$ Therefore, they have become an increasingly important tool in the study of single molecules and cell biophysics. For example, magnetic torque tweezers are especially suitable for torque measurement of DNA single molecule. ${ }^{2,3}$ By binding the magnetic particle to components of cellular membranes, the mechanosensitive ion channels can be modulated or controlled. ${ }^{4,5}$ In addition, the mechanical properties of cell membrane and cytoplasm can be analyzed by manipulating micro-magnetic probes. ${ }^{6-8}$ Recent advances in technique and novel magnetic particles' syntheses lead this tool to multi-functionality and expand its potential application. ${ }^{9}$

Superparamagnetic microbeads with a diameter from 0.5 to $5 \mu \mathrm{m}$ are usually used as measurement probes in magnetic tweezers. Via a magnetic gradient field, magnetic tweezers generate a force on the magnetic particles. In general, ideal magnetic tweezers should (1) be able to apply strong enough force, (2) have good maneuverability, and (3) have a structure applicable to biological experiments, e.g., cell culture. In order to generate sufficiently strong force, magnetic tweezers usually employ a structure with sharp-tipped magnetic poles and a

\footnotetext{
a) Author to whom correspondence should be addressed. Electronic mail: h.-j.krause@fz-juelich.de.
}

probe with high magnetization. For example, with magnetic beads with a diameter of $5 \mu \mathrm{m}$, single-pole electromagnetic tweezers can apply up to $100 \mathrm{nN}$ of pulling force at a distance of $10 \mu \mathrm{m}$ away from the pole. ${ }^{10}$ However, single-pole actuators can only apply magnetic forces in a single direction. By using multi magnetic poles, maneuverability can be achieved. With two opposing magnetic poles, bi-directional magnetic forces can be generated. ${ }^{11}$ With three or four poles symmetrically placed in one plane, 2D actuation can be achieved. ${ }^{12,13}$ To generate magnetic forces in 3D directions, a six-pole magnetic actuator, with three poles each in the top and bottom plane, has been designed and implemented. ${ }^{14,15}$ With the help of gravity force, 3D manipulation can also be achieved with poles only in one plane. ${ }^{16}$

Because of its simple structure, single pole magnetic tweezers are more straightforward and have been implemented more often. As shown in Ref. 10, analytical mathematical expressions can be used to accurately model the single pole electromagnet. For electromagnets with multiple poles, an approximate formula model can be applied when the magnetic force is small and the magnetic field changes smoothly in workspace. ${ }^{13,15}$ However, it is difficult to find a simple analytical model for 2D and 3D magnetic actuators with high force output because the magnetic force is generally a nonlinear function of position and currents of the multiple coils. In order to overcome this difficulty, we developed a new method to build a force model and an experimental setup. In this setup, a hexapole yoke made from soft magnetic materials with high permeability is used, which is compatible to both 2D and $3 \mathrm{D}$ actuations with different magnetic tip configurations. In this report, we will only focus on $2 \mathrm{D}$ actuation. The system 
we present here successfully combines high force output and good maneuverability.

In this paper, we present the design, modeling, implementation, and experimental results of a smart magnetic tweezers. This paper is organized as follows. Section II A briefly introduces the magnetic tweezers setup. The force model of magnetic bead actuation will be derived in detail in Sec. II B. In Sec. II C, the electronics design of the current amplifier is presented. Section II D covers optical tracking of the magnetic beads with different vision measurement algorithms. Based on these constituents, the feedback control is briefly presented in Sec. II E. The experimental results are given in Sec. III. Finally, the results are summarized and discussed in Sec. IV.

\section{MATERIALS AND METHODS}

\section{A. Magnetic tweezers setup}

In our magnetic tweezers setup shown in Figs. 1(a) and 1(b), the actuation parts include a main hexapole yoke and a fluidic cell with magnetic tips. The hexapole yoke is composed of 10 layers of laminated magnetic parts (PERMENORM ${ }^{\circledR}$ $5000 \mathrm{H} 2$, Vacuumschmelze GmbH \& Co. KG, Germany), which forms the main magnetic circuit. This soft magnetic material has a very small hysteresis, a high permeability at a large magnetization range, and a high saturation field of $1.55 \mathrm{~T}$. Every crisscrossing layer pattern is composed of 6 equal parts with a thickness of $0.5 \mathrm{~mm}$. These $\mathrm{Ni}-\mathrm{Fe}$ foils were isolated by a thin layer of varnish. Each magnetic pole of the main yoke has one actuating coil. Each coil has three taps to be configured with 80,160 , or 240 windings. In the central area, there is a small fluidic cell, which is fixed on the main yoke poles, as seen in Fig. 1(b). The fluidic cell includes 3 magnetic tips with $0.1 \mathrm{~mm}$ thickness (VACOFLUX ${ }^{\circledR} 50$, Vacuumschmelze $\mathrm{GmbH} \&$ Co. KG, Germany), which are fixed on a cover slip (0.3 $\mathrm{mm}$ thick, $18 \mathrm{~mm}$ diameter) using polydimethylsiloxane (PDMS, Sylgard 184, Dow Corning Corporation, USA). The tips do not contact the yoke and they are separated from it by the cover slip. For 3D actuation, the tips can be alternately placed at different height levels, similar to Ref. 14. The layout of the fluidic cell is presented in Fig. 1(c). A glass ring is placed on the tips to form a reservoir for the liquid sample. In order to avoid erroneous displacement during the process of fixing the tips, there is a supporting ring at the outside ends of tips. After curing of the PDMS, the ring is removed. In addition, different configurations of fluidic cells can be achieved easily by assembling them using individual tips. In the workspace between the tips, the magnetic beads can be manipulated, as shown in Fig. 1(c). All of the main yoke parts and tips in the fluidic cell were cut by laser to form the designed geometry and magnetically annealed under dry hydrogen atmosphere (manufactured by SEKELS GmbH, Germany). The light from a light-emitting diode (LED) cold-light source (CL 6000 LED, Carl Zeiss AG, Germany) is used to illuminate the workspace by an optical fiber from the bottom of the fluidic cell. With this, the heat contribution from light on the fluid cell is reduced, especially at high light intensity. In order to detect the particles, a USB 3.0 highspeed monochrome CMOS camera (MQ042MG-CM, XIMEA $\mathrm{GmbH}$, Germany) is used, which is specified to 90 frames per second at the full resolution of $2048 \times 2048$ pixel. $^{2}$ An

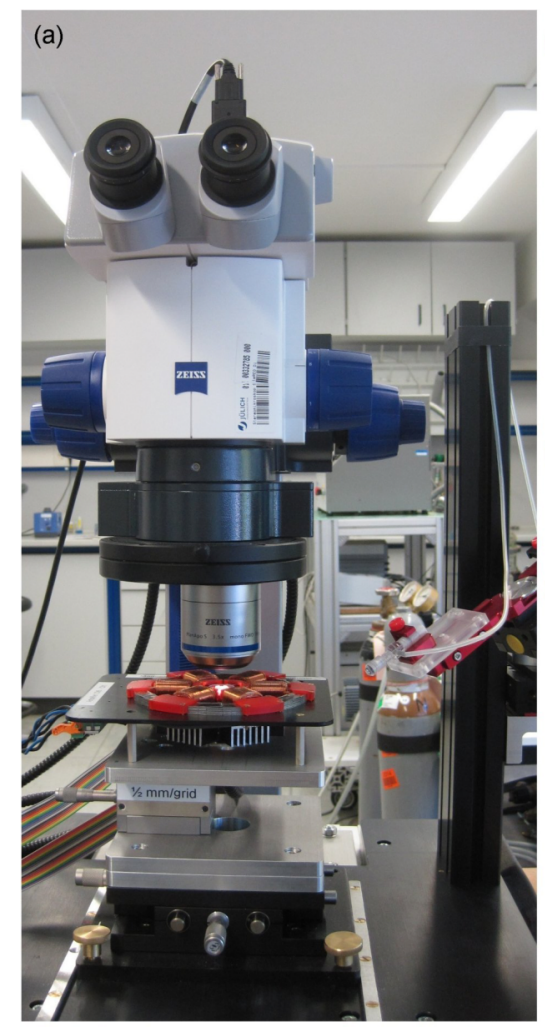

(b)

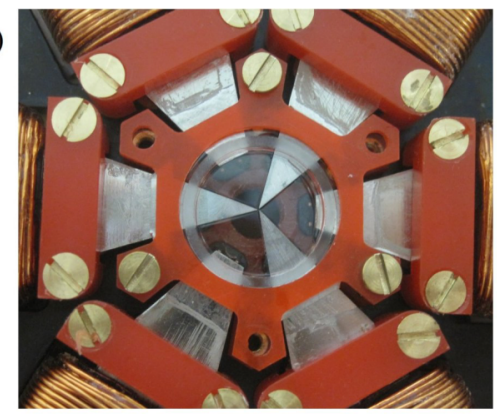

(c)

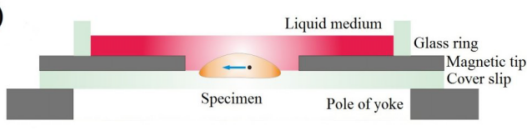

(d)

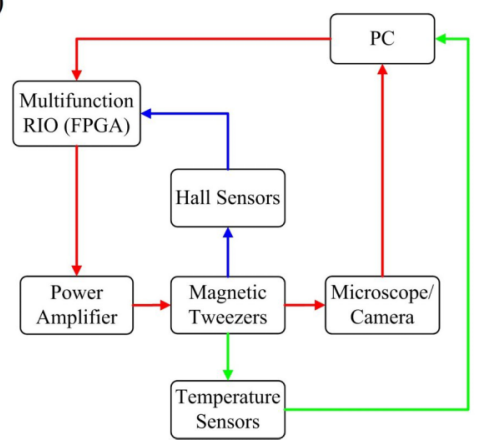

FIG. 1. (a) Setup of magnetic tweezers. (b) Top view of the central area of the main yoke, on which there is a fluidic cell with 3 magnetic tips made from 0.1 mm thick VACOFLUX ${ }^{\circledR} 50$ foil. (c) Layout of the fluidic cell. (d) Block diagram of the magnetic tweezers system. 
objective (Plan Apo S 3.5× mono, Carl Zeiss AG, Germany) with a large free working distance (FWD) of $16 \mathrm{~mm}$ is used with a stereo microscope (SteREO Discovery V8, Carl Zeiss AG, Germany). The magnetic tweezers is mounted onto an $\mathrm{XY}$ and $\mathrm{Z}$ stage (M-406 and MVN50, Newport Corporation, USA). It is well known that in a feedback control loop, the delays of the different blocks play an important role regarding the performance. Here, we used a multifunction reconfigurable input/output (RIO) card with an embedded fieldprogrammable gate array (FPGA) (PCIe-7841R, National Instruments Corporation, USA), which decreases the delay of output from computer to ca. $20 \mu$ s. In order to supply sufficiently high currents and to conveniently optimize the performance for the specific coils, we designed and built a multichannel power amplifier. The coils were wound using enameled copper wire with a diameter of $0.5 \mathrm{~mm}$. We also built a multi-channel magnetometer to help to calibrate the magnetic tweezers. Six small hall probes (A1302, Allegro MicroSystems, LLC, USA) were placed before the poles of main yoke underneath the top layers. In order to improve heat dissipation, a passive aluminum heat sink was mounted beneath the coils. The coil formers and the base plate for mounting the yoke are also made from aluminum. A multi-channel thermometer using six Pt100 temperature sensors was setup to individually monitor the temperature of each coil. The block diagram of our magnetic tweezers system is shown in Fig. 1(d). As the main part of system, the red feedback loop is used to actuate magnetic beads.

\section{B. Magnetic force and inverse force model}

\section{Magnetic field and force}

In our setup, the main yoke and fluidic cell form a closed magnetic circuit. The symmetrical tips in fluidic cell enclose a workspace where samples are located. The laminated pole tips driven by the main yoke are used to conduct and concentrate the magnetic flux into the workspace. With the ensuing magnetic field gradient, an actuation force can be applied on the magnetic beads. This magnetic force obeys ${ }^{17}$

$$
\mathbf{F}=(\mathbf{m} \cdot \nabla) \mathbf{B},
$$

where $\mathbf{B}$ is the magnetic flux density and $\mathbf{m}$ is the magnetic moment of the bead. As in Eq. (1), the force is proportional to the magnetic moment of the bead and to the magnetic field gradient at the bead's position. In general, the microbead used as probe is superparamagnetic. Its magnetic moment depends on external magnetic field, which can be approximately described by the Langevin function,

$$
\begin{gathered}
m(H)=m_{s}\left(\operatorname{coth}\left(\frac{H}{a}\right)-\frac{a}{H}\right), \\
\mathbf{B}=\mu_{r} \mu_{0} \mathbf{H} .
\end{gathered}
$$

Here, $\mathbf{H}$ refers to the magnetic field strength and $m_{s}$ is the saturated magnetic moment of the particle. Parameter $a$ is related to temperature and other physical properties. When the magnetic field is small, the magnetic susceptibility of the bead can be approximated as being constant. But when the magnetic field increases, the magnetic moment saturates very quickly. In formula (3), $\mu_{0}$ is the vacuum permeability and $\mu_{r}$ is relative permeability of medium which surrounds the particle. In air and water, $\mu_{r}$ is around 1.

One of the major goals of magnetic tweezers design is to achieve a strong force on the magnetic bead. From formulas (1) and (2), in the range of high field or high force, the gradient of magnetic field will play the main role to increase the force. In general, in order to obtain high magnetic field gradient, we can use a soft magnetic material with a high saturation magnetic field and adopt an optimized structure of magnetic poles. For this reason, the cobalt iron alloy VACOFLUX ${ }^{\circledR} 50$ is used for the magnetic tips, which has a very high saturation field of $2.33 \mathrm{~T}$. Besides, the sharp structure of tips and a small gap between tips can also enhance the magnetic field gradient. However, the distance among tips is a design variable and determined by the trade-off among the dimension of workspace, maximum amplitude and accuracy of magnetic force, manufacturing technique, and so on.

In order to optimize the geometrical design of the tips, we have inspected different structures using finite element simulation (COMSOL Multiphysics, COMSOL, Inc., USA). The simulations performed here were limited to $2 \mathrm{D}$ geometrical models in real dimension. The magnetic fields in the workspace were evaluated by nonlinear constitutive relation. The $B-H$ magnetization curves of the soft magnetic materials were adopted from the measurement results that the manufacturer obtained from our magnetic parts. Here, the shapes of all tips are like isosceles trapezoid, and the protrusion angles of the tips are around $35^{\circ}$. Unless otherwise indicated, the distance from the center of the workspace to the tip is $0.1 \mathrm{~mm}$, and each tip corner has a $10 \mu$ m radius. In order to decrease magnetic flux leakage, the sum of the three coil currents was always restricted to zero.

Because of the superparamagnetism of magnetic bead, the magnetic force always directs along the gradient of the magnitude of magnetic field, independent of the direction of the magnetic field. As shown in Fig. 2(a), with tips of $80 \mu \mathrm{m}$ in width, the saturation magnetic field (magnitude) and corresponding magnetic force in workspace were obtained from simulation at currents: 174 A turns on right source coil; -87 A turns on two other counter coils. Here, all the force calculations were based on the magnetic moment of Dynabeads ${ }^{\circledR}$ M-270 Carboxylic Acid (Thermo Fisher Scientific, Inc., USA). Unless otherwise stated, we abbreviate these beads as M-270 in this paper. In the area indicated by a black square $(1 / 2$ area, $0.1 \mathrm{~mm} \times 0.1 \mathrm{~mm})$ in Fig. 2(a), the force is relative homogeneous, which is important for control. The center of workspace is marked as $\mathrm{O}$, with a force of $1219 \mathrm{pN}$ along the $x$ axis. Because the right tip is located close by, the force at point $\mathrm{B}$ is maximum in the upper half square, with $|\mathrm{F}(\mathrm{B})| /|\mathrm{F}(\mathrm{O})|=1.24$. In the area between corners of tips, despite a high magnetic field, the magnetic force is low because of small magnetic field gradients. So, the minimum force in the upper half square is located at $\mathrm{A}$, with $|\mathrm{F}(\mathrm{A})| /|\mathrm{F}(\mathrm{O})|=0.69$. The ratio $\mathrm{Fy} / \mathrm{Fx}$ is the highest at point $\mathrm{C}$, namely, 0.69. Here, too high a ratio Fy/Fx means the force component directing outward from the left-up tip is low. So it is hard to actuate the bead in the nearby area. According to the symmetry, there are similar features in the bottom half 
(a)

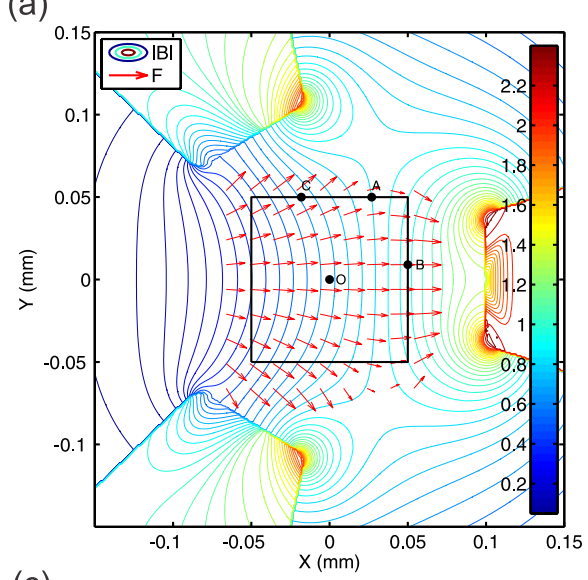

(c)

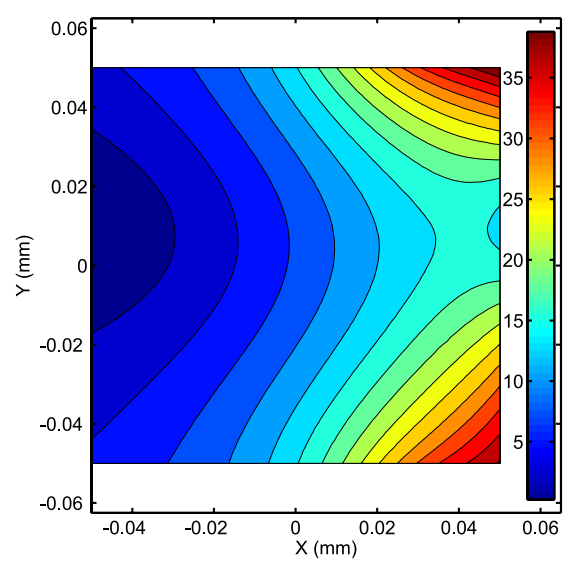

(b)

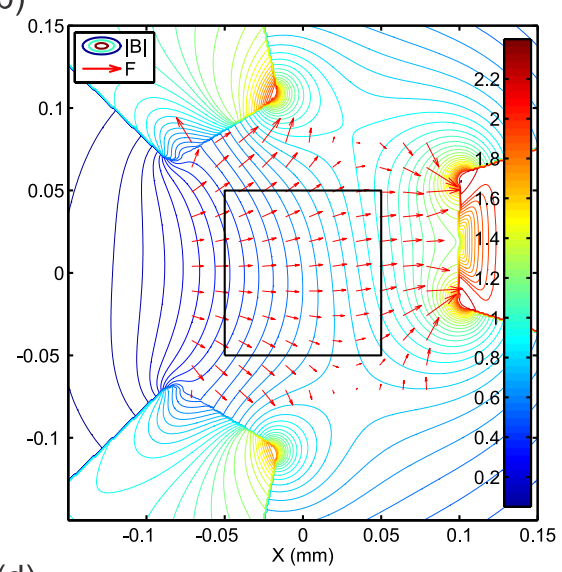

(d)

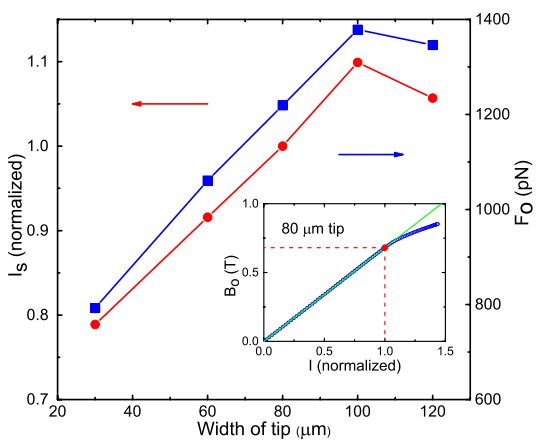

FIG. 2. (a) Simulated saturation magnetic field (magnitude) and force with $80 \mu \mathrm{m}$ wide tips. (b) Simulated saturation magnetic field (magnitude) and force field with $80 \mu \mathrm{m}$ wide tips, and the right tip has a displacement of $20 \mu \mathrm{m}$ along $y$ axis. (c) Simulated force error (in percent) of the displaced tip geometry in (b) vs. the symmetric tip geometry in (a). (d) Simulated forces at workspace center $(\mathrm{O})$ and saturation currents for different width of tips. The dependence of the magnetic field at $\mathrm{O}$ on the current for $80 \mu \mathrm{m}$ tips is presented in the inset figure. The saturation currents are normalized by the saturation current of $80 \mu \mathrm{m}$ tips (174 A turns, as indicated by the red dot in the inset figure).

square. Outside of this square, the force changes rapidly. Although higher force can be achieved when approaching the tips, the resultant higher anisotropy will also deteriorate the maneuverability. Especially, there are some "dead zones" near the tips, where the particles are always pulled toward the tips at any nonzero input currents. Due to the limited accuracy of the manufacturing process, some tips may exhibit deformations. In order to inspect this effect, the right tip was shifted in the simulation by $20 \mu \mathrm{m}$ along the $y$ axis, as shown in Fig. 2(b). It is obvious that there is a large change of the magnetic field distribution in the area near the right tip. The error percentage of force induced by this deformation is depicted in Fig. 2(c) as a surface plot of the 1/2 area. The error is highest at the upper and lower corners towards the right tip. However, the error decreases with distance from the deformed tip. Within the inner $1 / 4$ area $(0.05 \mathrm{~mm} \times 0.05 \mathrm{~mm})$, the error is less than $18.7 \%$. But the maximum error increases to $41.4 \%$ in $1 / 2$ area. In order to optimize the width of the tip, we have simulated tips with widths of $30,60,80,100$, and $120 \mu \mathrm{m}$. The results are presented in Fig. 2(d). With a wider tip, the saturation current increases; hence, higher magnetic flux can be applied to the workspace, which also enhances the force. When the tip has a width of $100 \mu \mathrm{m}$, which equals the distance from the central point to the tip, a maximum force of $1378 \mathrm{pN}$ can be exerted on a single M-270 bead. This result agrees well with the findings presented in Ref. 12.

\section{Inverse force model}

From Fig. 2(a), it is obvious that the force is highly position dependent. In order to generate an accurate force on the bead in the workspace, an inverse force model is developed. With this inverse force model, we can determine the input currents that produce the desired magnetic force. First, based on the simulation result, a lookup table (LUT) of coil currents to magnetic field was obtained. As shown in the inset plot of Fig. 2(d), below magnetic saturation, the linearity between $B$ and $I$ is very good. Moreover, because of the symmetry of the magnetic poles, we can compose the final magnetic field by rotating and then superimposing the field generated by each coil current, as in formula (4),

$$
\left\{\begin{array}{c}
\mathrm{B}_{\mathrm{x}}(x, y)=\sum_{p} q_{p}^{x}(x, y) I_{p}, \\
\frac{\partial \mathrm{B}_{\mathrm{x}}}{\partial x}(x, y)=\sum_{p} r_{p}^{X x}(x, y) I_{p} .
\end{array}\right.
$$

Here, $q_{p}$ and $r_{p}$ are position dependent parameters and index $p$ is the number of the magnetic pole. In practice, parameters $q_{p}$ 
and $r_{p}$ are adopted as a LUT with $2 \mu \mathrm{m}$ grid spacing. With this LUT, the magnetic field of any position in work space is bilinearly interpolated using the four nearest neighbor grid points.

As in formula (2), the magnetic moment of the bead can be described by a Langevin function. So the force components are coupled by this nonlinear function, of which it is difficult to obtain an analytic solution. In order to find a direct way to compose any desired force vector, we adopted a piece-wise linear approximation to describe $\mathbf{m}(\mathbf{B})$ of the magnetic bead, as in formula (5),

$$
\mathbf{m}(|\mathbf{B}(\mathrm{x}, \mathrm{y})|)=k_{i}|\mathbf{B}(\mathrm{x}, \mathrm{y})|+b_{i} \text {, when } \mathrm{B}_{i} \leq|\mathbf{B}(\mathrm{x}, \mathrm{y})|<\mathrm{B}_{i+1} .
$$

The space between boundary points $B_{i}$ is chosen based on the slope change of $\mathbf{m}(\mathbf{B})$. So, the accuracy of the piecewise linear magnetization function can be improved easily by increasing the number of pieces. In this work, we used 18 pieces to characterize the magnetic bead. As shown in Fig. 3, a piecewise linear magnetization function can characterize the magnetization of a bead very well with few segments. The data of M-270 beads supplied by the vendor are quoted as magnetic susceptibility: $6 \times 10^{-4} \mathrm{~m}^{3} / \mathrm{kg}$, saturation magnetization: $13 \mathrm{~A} \cdot \mathrm{m}^{2} / \mathrm{kg}$, density: $1.6 \mathrm{~g} / \mathrm{cm}^{3}$, and diameter: $2.8 \mu \mathrm{m}$. The results of a fit to a Langevin function are $\mu_{0} \cdot a=0.021$ and $m_{s}=2.23 \times 10^{-13} \mathrm{~A} \cdot \mathrm{m}^{2}$.

By combining (1), (4), and (5), we can obtain

$$
\left\{\begin{array}{c}
\mathrm{F}_{x}(\mathrm{x}, \mathrm{y})=\left(\frac{b_{i}}{|\mathbf{B}(\mathrm{x}, \mathrm{y})|}+k_{i}\right) \sum_{p, p^{\prime}}\left(q_{p}^{x}(\mathrm{x}, \mathrm{y}) r_{p^{\prime}}^{X x}(\mathrm{x}, \mathrm{y}) I_{p} I_{p^{\prime}}+q_{p}^{y}(\mathrm{x}, \mathrm{y}) r_{p^{\prime}}^{Y x}(\mathrm{x}, \mathrm{y}) I_{p} I_{p^{\prime}}\right) \\
\mathrm{F}_{y}(\mathrm{x}, \mathrm{y})=\left(\frac{b_{i}}{|\mathbf{B}(\mathrm{x}, \mathrm{y})|}+k_{i}\right) \sum_{p, p^{\prime}}\left(q_{p}^{x}(\mathrm{x}, \mathrm{y}) r_{p^{\prime}}^{X y}(\mathrm{x}, \mathrm{y}) I_{p} I_{p^{\prime}}+q_{p}^{y}(\mathrm{x}, \mathrm{y}) r_{p^{\prime}}^{Y y}(\mathrm{x}, \mathrm{y}) I_{p} I_{p^{\prime}}\right) \\
|\mathbf{B}(\mathrm{x}, \mathrm{y})|=\sqrt{\mathrm{B}_{\mathrm{x}}(\mathrm{x}, \mathrm{y})^{2}+\mathrm{B}_{\mathrm{y}}(\mathrm{x}, \mathrm{y})^{2}} \\
\sum_{p} I_{p}=0 .
\end{array}\right.
$$

Based on Eqs. (4)-(6), it is easy to solve for the currents from a desired force in a recursive way. By iterating through the linear sections starting from the first section with $k_{0}$ and $b_{0}$, it is checked if the variable $\mathbf{B}$ resolved from Eqs. (6) and (4) also satisfies Eq. (5). If yes, this result is the solution; if not, the iteration is continued. For quadratic equations, there are usually two solutions. Here, we adopted the smaller one to keep currents as low as possible.

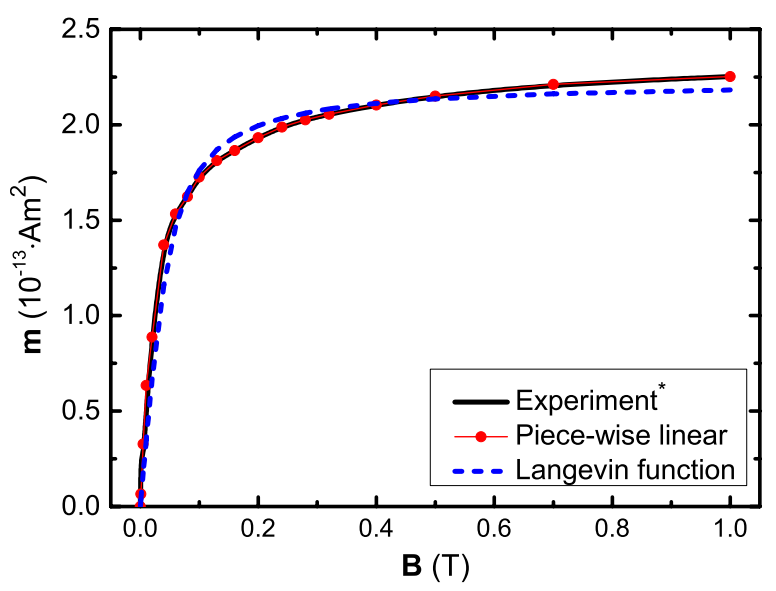

FIG. 3. Magnetization of a single M-270 bead. (Asterisk denotes that the experimental data are quoted from the vendor.)

\section{Electronics}

Due to the high permeability of PERMENORM ${ }^{\circledR}$, the main yoke coil with 240 turns exhibits a high inductance of $8.86 \mathrm{mH}$ at $100 \mathrm{~Hz}$, which was measured by LCR meter (ST2826A, Sourcetronic GmbH, Germany). When the frequency goes up, the inductance of the coil decreases a little, for example, to $2.67 \mathrm{mH}$ at $224.5 \mathrm{kHz}$. Around $300 \mathrm{kHz}$, there is a sharp drop in inductance and the dissipation factor increases at the same time. It is a challenge to drive these high inductance coils fast and accurately. When using a normal amplifier to drive such high inductive load, it is very easy to cause oscillation. In this work, in order to apply a fast and stable force, we custom built a multi-channel power amplifier. High power operational amplifiers OPA541 (Texas Instruments, Inc., USA) were used which have a maximum dissipation power of $125 \mathrm{~W}$. A high-speed precision operational amplifier OPA602A (Texas Instruments, Inc., USA) was employed as voltage-to-current conversion amplifier. Besides, two primary switched power supplies of 1200 W each (EXW 24.50 installation unit, Kniel GmbH, Germany) were adopted. With these, the fluctuation of the current output is limited to less than $1 \mathrm{LSB}$ of the 16 bit DAC, corresponding to $150 \mu \mathrm{A}$. With up to three specific stability compensation circuits for inductive loads, the amplifier was optimized to achieve a good performance for each coil configuration (80, 160, and 240 turns). 

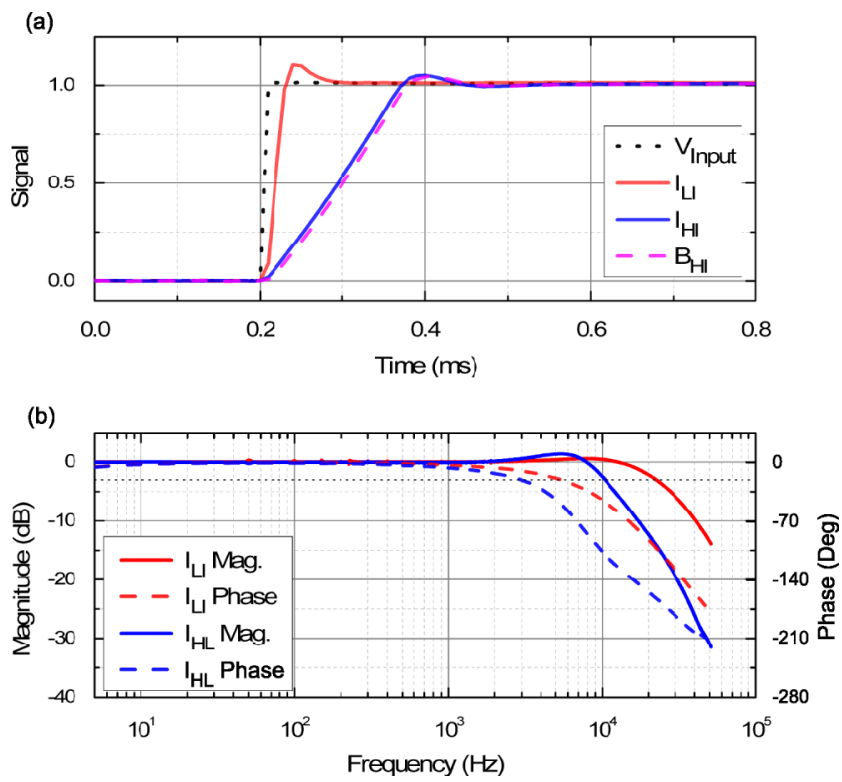

FIG. 4. (a) Step response of coil current and magnetic field at low inductance ( 80 windings) and high inductance ( 240 windings) coil configurations. The input signal voltage is $1 \mathrm{~V}$. For clarity, the current $(0.5 \mathrm{~A})$ and magnetic field $(147 \mathrm{G})$ are normalized to 1 . (b) Frequency response of amplifier at different coil configurations.

The step response of current and magnetic field upon an input signal of $1 \mathrm{~V}$ is shown in Fig. 4(a). The rise time of the current is less than $50 \mu \mathrm{s}$ for the low inductance coil (80 turns) and $200 \mu$ s for the high inductance coil (240 turns). The maximum overshoot is less than $10 \%$. All of these signals were acquired synchronously by a DAQ card (NI USB6259, National Instrument Corporation, USA) at $100 \mathrm{kHz}$. The frequency response is presented in Fig. 4(b), which was measured by a dynamic signal analyzer (HP 35670A, Hewlett Packard, USA). The bandwidth is $22.4 \mathrm{kHz}$ for the low inductance coil and $10.4 \mathrm{kHz}$ for the high inductance coil. The respective phase margins are $72.6^{\circ}$ and $70^{\circ}$.

Because forces are directly dependent on magnetic field, a magnetometer based on hall probes was built to monitor the magnetic flux through the core. The probes were attached at the ends of the main yoke poles. As shown in Fig. 4(a), the magnetic field can follow the coil current very well. The magnetic field step response for the low inductance coils is not shown because it is limited by the bandwidth of the Hall sensors $(20 \mathrm{kHz})$. With these sensors, no hysteresis was observed, which we ascribe to the low coercivity $(3.41 \mathrm{~A} / \mathrm{m})$ and high permeability of yoke material $\left(\mu_{\mathrm{r}}>8000\right.$ up to 1 $\mathrm{T})$. Between experiments, the magnetization of the main yoke was degaussed by applying sinusoidal currents with rotating decaying amplitude, which quench the residual magnetism in every part of the soft-magnetic yoke.

In order to protect the coils from overheating, we mounted a heat sink under the coils. Moreover, a six channel thermometer was built to monitor the temperature of the coils. Thanks to the heat sink and the aluminum mechanical framework, even with continuous 4 A current lasting for 10 min, the highest temperatures measured at coil, heat sink, and fluidic cell were $112.5^{\circ} \mathrm{C}, 78.0^{\circ} \mathrm{C}$, and $35.5^{\circ} \mathrm{C}$, respectively. Before this measurement, $0.25 \mathrm{ml}$ milli-Q water was put in the fluidic cell, and then the temperature sensor was immersed in water.

\section{Particle tracking}

In order to determine the position and to measure the velocity of microbeads, we implemented a video based particle tracking system, which allows for high flexibility and is easy to implement. This system includes a vision acquisition and analysis software and a fast speed digital camera with the microscope. With a small region of interest (ROI) of $100 \times 100$ pixel, ${ }^{2}$ our camera can capture more than 2000 frames per second. The speed limiting factor for small ROIs is the rate at which data can be read off the pixel sensor. The vision acquisition and analysis software was developed in LabVIEW (National Instruments, USA). All vision analysis was done on a multicore CPU (Core i7 3770K, Intel Corporation, USA). In order to obtain a good image quality especially at a short exposure time situation, the fluidic cell was illuminated in transmission mode. By a calibration slide, we determined the scale of our imaging system to $198 \mathrm{~nm} /$ pixel. In this work, we have implemented three position tracking algorithms for different application conditions, as given in Table I.

In the center of mass (COM) algorithm, the profile of the microbead is first detected by a threshold. Then, the central position of the microbead is calculated based on the weighted pixel value. ${ }^{18}$ Because of simple calculation, this method is very fast. However, this method can only be used for good optical conditions with a clear contrast between particle and background. Due to its invariance to linear brightness and contrast variations, normalized cross correlation (NCC) has found numerous applications in image processing, such as pattern recognition and template matching. Here, we track the bead by correlating the current image with a template image of bead, which can effectively eliminate background disturbances like in Ref. 19. The template image is built by interpolating along radial direction with mean intensity profile along four radial directions $x-, x+, y-$, and $y+$. Before every experiment, the template image of bead was manually selected by an annulus tool. The exact position along $x$ and $y$ directions is fit with a second-order polynomial using five points around the maximum value in the result of NCC. In general, the $\mathrm{NCC}$ is more robust and has a higher resolution than COM, especially in a noisy image. ${ }^{18,20}$ Although NCC has high accuracy, the computational effort required is much higher. In order to increase the speed, we implemented this algorithm in parallel computing. Besides, we also used optical flow (OF) method, which is based on pyramid-based Lucas and Kanade

TABLE I. Particle tracking algorithms.

\begin{tabular}{llll}
\hline \hline Algorithm & \multicolumn{1}{c}{ Speed $^{\mathrm{a}}$} & Resolution & \multicolumn{1}{c}{ Comment } \\
\hline Center of mass & Fast $(6520 \mathrm{~Hz})$ & Medium & $\begin{array}{l}\text { Sensitive to image } \\
\text { quality }\end{array}$ \\
Cross correlation & Slow $(991 \mathrm{~Hz})$ & High & $\begin{array}{l}\text { Robust } \\
\text { Optical flow }\end{array}$ \\
Medium $(2870 \mathrm{~Hz})$ & Low & Multi-object tracking \\
\hline
\end{tabular}

${ }^{a}$ The speed values are given as typical values for single bead tracking with an image size of $200 \times 200 \mathrm{pixel}^{2}$, the size of normalized cross-correlation (NCC) template image is $30 \times 30 \mathrm{pixel}^{2}$, and the search window of optical flow (OF) algorithm is $60 \times 60 \mathrm{pixel}^{2}$. 
algorithm as implemented in the Vision and Motion module of LabVIEW. With this method, it is easy to track multiple objects even when they overlap on the image. However, the resolution of this algorithm is low, and the absolute error will accumulate continuously. In order to shorten the time for particle tracking, we adopted a moving sub-ROI for all the three algorithms, so that only the pixels in this region were analyzed. In addition, for convenience of post-processing, we also implemented a circular buffer to queue the images from camera and compress them into video in a parallel process. In practice, due to the limited speed of CPU, only part of the experimental images can be archived (for example, one out of three at a ROI of $600 \times 600$ pixel $^{2}$ ).

\section{E. Feedback control}

As mentioned in Sec. II B 1, the magnetic particle is always attracted towards a magnetic pole, so there is no stable state at which the particle can remain with constant currents applied. In order to stabilize and control the motion of the magnetic particle, we implemented an active feedback controller. With the inverse force model deduced as previous, we can linearize the force output of magnetic tweezers. As a result, the controller design was greatly simplified. The remaining linear dynamics of the system can thus be handled with a linear controller. In order to decrease the steady state error, a proportional integral (PI) controller was deployed. Using Simulink/Matlab (MathWorks, Inc., USA) with trial-and-error design method, the parameters of the PI controller were determined. Then, this continuous controller was converted to a digital controller by using the trapezoid rule. Moreover, when the output of this controller reached some set value, the integrator part was reset to avoid large overshoot (anti-windup). ${ }^{21}$

A detailed analysis on the effect of delay can be found in Ref. 16. As the speed strongly influences the positioning accuracy and maneuverability, ${ }^{13}$ we tried to improve it as much as possible. Thanks to the high speed of the camera, the fast particle tracking code and the very low delay of RIO card, the speed of actual control loop was $303 \mathrm{~Hz}$ at ROI of $600 \times 600$ pixel $^{2}$ and $870 \mathrm{~Hz}$ at $200 \times 200$ pixel $^{2}$ with COM tracking algorithm. Faster speeds than $1 \mathrm{kHz}$ can be achieved easily by adopting a smaller ROI.

\section{EXPERIMENTAL VERIFICATION AND ANALYSIS}

In order to verify our model, the magnetic force was calibrated using the classical viscous drag approach. The force applied on bead is measured by observing the viscous drag at constant coil current configuration. According to Stokes' formula, the force $\mathbf{F}$ on a bead with hydrodynamic radius $r$ moving with velocity $\mathbf{v}$ in a fluid with viscosity $\eta$ is given by

$$
\mathbf{F}=6 \pi \eta r \mathbf{v}
$$

In Newtonian fluid approximation, the viscosity can be regarded as a constant. The velocity of a bead moving through a viscous fluid is proportional to the force. In the calibration experiments, we used the $2.8 \mu \mathrm{m}$ magnetic beads M-270 with carboxylic acid group shell. The measurements were performed in fluids with a low and a high viscosity. The first fluid with low viscosity was diethylene glycol (DEG, puriss, $99.0 \%$, Sigma-Aldrich, USA), which was mixed with the M270 solution at a ratio of 200:1. The viscosity of DEG was $31.4 \pm 0.16 \mathrm{mPa} \cdot \mathrm{s}$ at $23^{\circ} \mathrm{C}$, as measured by a viscosity meter (microVISC, RheoSense, Inc., USA). The highly viscous fluid was made from $85 \%$ glycerol (99.0\%, Alfa Aesar GmbH, Germany) mixed with $15 \%$ water. The ratio of mixed fluid with bead solution was 400:1. Before every experiment, 0.2 $\mathrm{ml}$ of the mixing solution was injected into the fluidic cell with pipette. After 10 min of sedimentation, drag experiments were done. In this work, all experiments were done with the same fluidic cell, which had a $0.1 \mathrm{~mm}$ radius of workspace, $60 \mu \mathrm{m}$ width of tip. The particle tracking algorithm used here was NCC.

As in Fig. 5(a), the fluidic cell was aligned manually under microscope by adjusting the XY stage with the help of the overlaying of design tips contour. As we can see, the actual tips were not very well defined. All tips have a smaller width than desired, especially the tip at bottom right. Also the top right tip was much thinner than designed. A bead was positioned near the center of the workspace. In order to

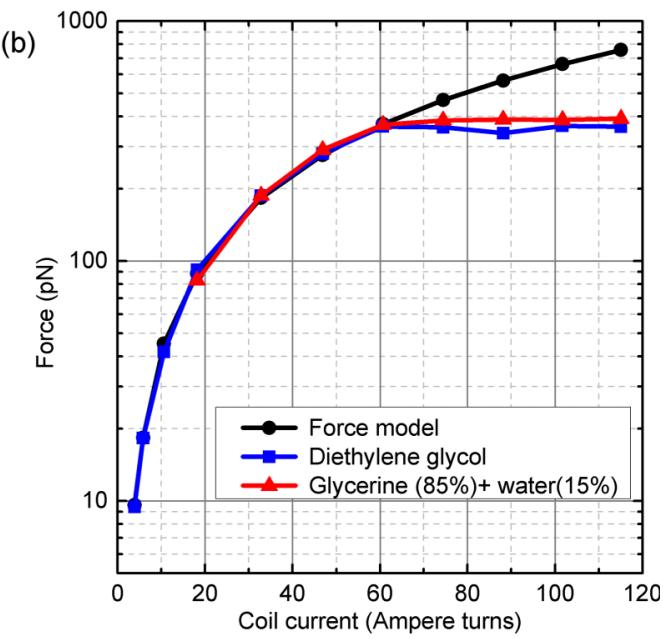

FIG. 5. (a) Fluidic cell and beads. In the central workspace, there are several spots in PDMS. (b) Calibration of force at center of workspace. 
(a)

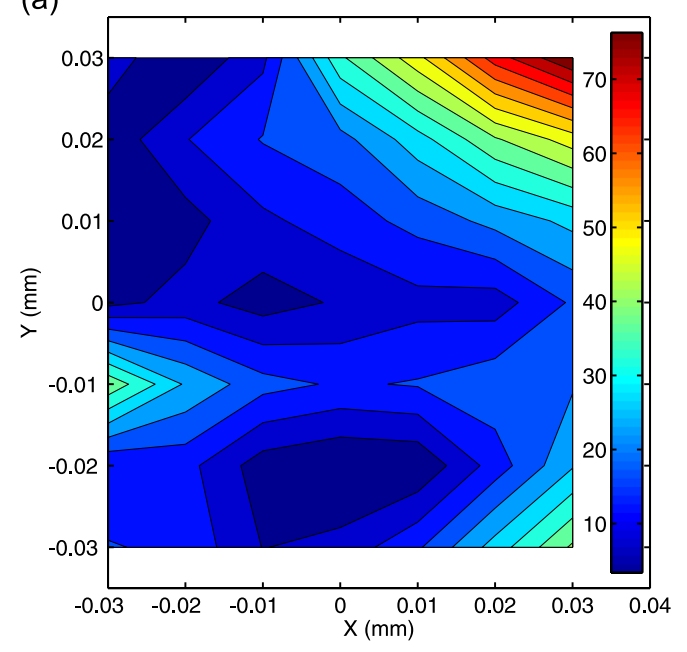

(b)

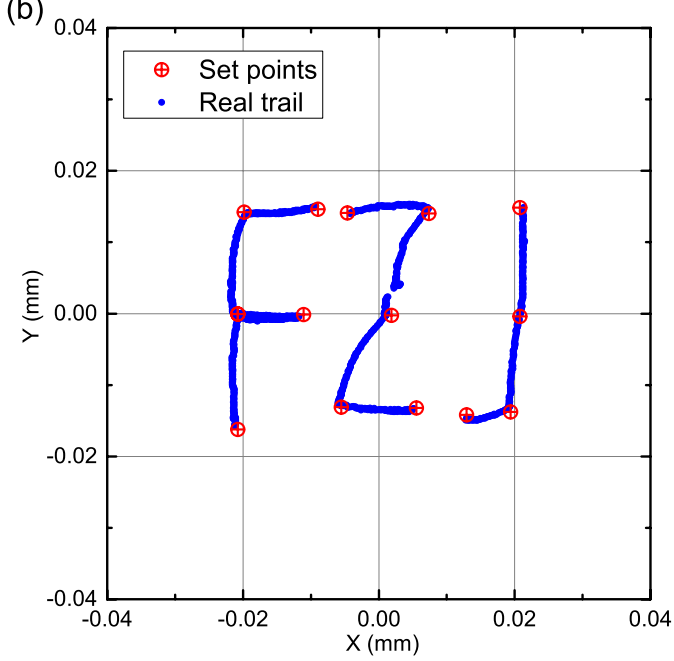

FIG. 6. (a) Force error (percent) between experiment and force model was mapped at grid $7 \times 7$ with step of $10 \mu \mathrm{m}$. (b) Bead was actuated to move along predefined set points in DEG. For the clarity, the intermediate segments between letters were not shown.

calibrate the force, we dragged the bead along $x$ direction in the central area from $(-15 \mu \mathrm{m}, 0 \mu \mathrm{m})$ to $(15 \mu \mathrm{m}, 0 \mu \mathrm{m})$. Before every measurement, the bead was actuated to the start point $(15 \mu \mathrm{m}, 0 \mu \mathrm{m})$ by the PI controller. Then, the controller was turned off and the bead was actuated by constant input currents. The movements of bead were tracked until the bead arrived at the final point $(-15 \mu \mathrm{m}, 0 \mu \mathrm{m})$. After that, the PI controller was activated and the bead was actuated towards the start point again. Only the tracking points between $-10 \mu \mathrm{m}$ and $10 \mu \mathrm{m}$ were analyzed. The velocity was deduced by fitting the position as a function of time with a linear function. The force acting on the bead during the constant current-on phase was then computed from the velocities. The magnetic force on a bead that was held at the central point was compared to the force module as a function of the coil current. The results are presented in Fig. 5(b). As the current through the driving coils increases, the applied magnetic force increased as predicted by the model. Considering the actual shape of tips, the experimental force agrees well with the force model in the low force range $(<400 \mathrm{pN})$. When the set force was larger than $400 \mathrm{pN}$, some fluctuations were observed in practical force output, which are probably due to the high speed of the bead. In order to clarify this, we used another fluid of $85 \%$ glycerol with higher viscosity for this high force measurement. The viscosity of $85 \%$ glycerol was deduced by the DEG result at low force $(100-300 \mathrm{pN})$. With this fluid, the bead shows a clear saturation behavior towards $360 \mathrm{pN}$. Several causes can be ascribed to this early saturation: (1) the width of tips is smaller than the design and the simulation model; (2) the distance between tips is larger than the design and the simulation model; and (3) perhaps most important, due to laser cutting, the effective total "saturation" magnetic field in the outside layer of the tips decreases. The maximum experimental force is about $400 \mathrm{pN}$, less than the simulated value $(1060 \mathrm{pN})$. Thanks to the sharp structure and the high permeability of the tips, as in simulation, just a very low current is sufficient to achieve a high force.

In order to evaluate the effect of tip deformation, we also measured the force distribution on a $7 \times 7$ grid $(x$ : from
$-30 \mu \mathrm{m}$ to $30 \mu \mathrm{m}$ and $y$ : from $-30 \mu \mathrm{m}$ to $30 \mu \mathrm{m}$, each with $10 \mu \mathrm{m}$ step) in DEG. The bead was first trapped at each starting location by active feedback control, and then the drag measurements were done. The response of the probe's motion was also analyzed by linear fit. Here, the force model was activated in drag experiment to set a constant force $20 \mathrm{pN}$ along $x$ direction at each grid point. The force error map is shown in Fig. 6(a). The error was calculated by the formula $100 \% \times\left(\mathrm{F}_{\text {exp }}-\mathrm{F}_{\mathrm{mod}}\right) / \mathrm{F}_{\text {mod. }}$. As in simulation, in the central area and far away from deformed tips, the errors are small. It is obvious that the error in upper right area is high. We inferred this high error from the thinner tip. In addition, in order to inspect the feedback control, we actuated the magnetic bead to follow a predefined trajectory in $1 / 4$ area by few set points, as shown in Fig. 6(b). Here, the output force of PI controller was limited to $30 \mathrm{pN}$ in both $x$ and $y$ directions. When the bead got closer than $0.5 \mu \mathrm{m}$ to one set point, then it was actuated towards the next set point. In the trail of "F," there is a little bending towards the left in the vertical line. Similar behavior was observed in the slash of "Z." This may result from the unbalance of the tips. The force component from the left tip is higher than desired. Although there are several spots in the workspace as shown in Fig. 5(a), the bead can be tracked very well by NCC algorithm (COM did not work in this situation). Some tracking points were missed at the center of the " $Z$." This is because of a spot in that area with a similar optical pattern as the bead, which made the polynomial fits of cross correlation fail.

\section{SUMMARY AND DISCUSSION}

Both high force output and good maneuverability are achieved with our newly developed magnetic tweezers. Up to $400 \mathrm{pN}$ can be applied on a $2.8 \mu \mathrm{m}$ bead in any direction within a plane. Active video tracking based feedback control is implemented at speed up to $1 \mathrm{kHz}$. An inverse model which can deal with high force and nonlinearities was derived to facilitate the implementation of feedback control. High 
force can be generated in a large workspace with size of $0.1 \times 0.1 \mathrm{~mm}^{2}$.

Both simulation and experiment indicated that the shape of tips has a strong effect on the magnetic force. Because of the difference between actual tips shape and design, the experimental saturation force is about half of the simulation value. Although we can increase the maximum magnetic force by using a smaller workspace, it may deteriorate the accuracy of force (maneuverability). In practice, it should be a tradeoff between maximum force and the accuracy of force. However, both the potential maximum force and maneuverability could be improved by optimizing the manufacturing process of magnetic tips. In addition, thanks to the high permeability of main yoke and tips, the bandwidth of the amplifier can be enhanced very easily by using a coil with fewer turns. According to the findings in Ref. 20, with a higher magnification objective (for example, water immersion with high numerical aperture), the spatial resolution could also be improved.

\section{ACKNOWLEDGMENTS}

We thank Philipp Rinklin for beneficial discussion about the experiment, Norbert Wolters for building the electronics, Dieter Lomparski for help in LabVIEW software and mechanical structures design, Alexander Kuck and his colleagues who fabricated the mechanical structures, and Dr. Swen Graubner (SEKELS GmbH) for help with the magnetic parts design and manufacturing.
${ }^{1}$ K. C. Neuman and A. Nagy, Nat. Methods 5, 491 (2008).

${ }^{2}$ A. Celedon, I. M. Nodelman, B. Wildt, R. Dewan, P. Searson, D. Wirtz, G. D. Bowman, and S. X. Sun, Nano Lett. 9, 1720 (2009).

${ }^{3}$ J. Lipfert, J. W. J. Kerssemakers, T. Jager, and N. H. Dekker, Nat. Methods 7, 977 (2010).

${ }^{4}$ J. Dobson, Nat. Nanotechnol. 3, 139 (2008)

${ }^{5}$ J.-H. Lee, J.-W. Kim, M. Levy, A. Kao, S.-H. Noh, D. Bozovic, and J. Cheon, ACS Nano 8, 6590 (2014).

${ }^{6}$ H. Huang, C. Y. Dong, H.-S. Kwon, J. D. Sutin, R. D. Kamm, and P. T. C. So, Biophys. J. 82, 2211 (2002).

${ }^{7}$ A. R. Bausch, W. Möller, and E. Sackmann, Biophys. J. 76, 573 (1999).

${ }^{8}$ L. Ramms, G. Fabris, R. Windoffer, N. Schwarz, R. Springer, C. Zhou, J. Lazar, S. Stiefel, N. Hersch, U. Schnakenberg, T. M. Magin, R. E. Leube, R. Merkel, and B. Hoffmann, Proc. Natl. Acad. Sci. U. S. A. 110, 18513 (2013).

${ }^{9}$ D. Kilinc and G. U. Lee, Integr. Biol. 6, 27 (2014).

${ }^{10} \mathrm{P}$. Kollmannsberger and B. Fabry, Rev. Sci. Instrum. 78, 114301 (2007).

${ }^{11}$ B. G. Hosu, K. Jakab, P. Bánki, and F. I. Tóth, Rev. Sci. Instrum. 74, 4158 (2003).

${ }^{12}$ A. H. B. de Vries, B. E. Krenn, R. van Driel, and J. S. Kanger, Biophys. J. 88, 2137 (2005).

${ }^{13}$ Z. Zhang, Y. Huang, and C.-H. Menq, IEEE Trans. Rob. 26, 531 (2010).

${ }^{14}$ J. K. Fisher, J. Cribb, K. V. Desai, L. Vicci, B. Wilde, K. Keller, R. M. Taylor, J. Haase, K. Bloom, E. T. O’Brien, and R. Superfine, Rev. Sci. Instrum. 77, 023702 (2006).

${ }^{15}$ Z. Zhang and C.-H. Menq, IEEE/ASME Trans. Mechatronics 16, 421 (2011).

${ }^{16}$ C. Gosse and V. Croquette, Biophys. J. 82, 3314 (2002).

${ }^{17}$ T. H. Boyer, Am. J. Phys. 56, 688 (1988).

${ }^{18}$ M. K. Cheezum, W. F. Walker, and W. H. Guilford, Biophys. J. 81, 2378 (2001).

${ }^{19}$ J. Lipfert, J. J. W. Kerssemakers, M. Rojer, and N. H. Dekker, Rev. Sci. Instrum. 82, 103707 (2011).

${ }^{20}$ M. T. J. van Loenhout, J. W. J. Kerssemakers, I. De Vlaminck, and C. Dekker, Biophys. J. 102, 2362 (2012).

${ }^{21}$ G. F. Franklin, J. D. Powell, and A. Emami-Naeini, Feedback Control of Dynamic Systems, 3rd ed. (Addison-Wesley Publishing Company, Inc., USA, 1995), p. 196. 